\title{
Plasma and fiber spatial multi-mode initiated stable soliton self-compression and spectral bouncing in air-filled Kagome HCPCF
}

\author{
M. Maurel ${ }^{1,2}$, F. Amrani ${ }^{1,2}$, Ihar Babushkin ${ }^{3,4}$, B. Debord ${ }^{1,2}$, F. Gérôme ${ }^{1,2}$ and F. Benabid ${ }^{1,2}$ \\ ${ }^{1}$ GPPMM Group, Xlim research institute, University of Limoges, 123 avenue Albert Thomas 87060 Limoges, FRANCE. ${ }^{2}$ Glophotonics, 123 \\ avenue Albert Thomas 87060 Limoges, FRANCE. ${ }^{3}$ Institut Institute of Quantum Optics, Leibnitz Hannover University, Hannover, Germany. \\ ${ }^{4}$ Cluster of Excellence PhoenixD Welfengarten 1, 30167 Hannover, Germany.f.benabid@xlim.fr
}

Abstract: We report on a nonlinear compression down to 20 fs in air-filled HCPCF. Novel spectraltemporal dynamic is demonstrated. Spectral bouncing of the compressed pulse is triggered by the interplay between Raman redshift and plasma blueshift.

The recent combination of Inhibited-Coupling hollow-core photonic crystal fiber (IC-HCPCF) and high-energy ultrashort optical pulses (HE-USP) proved to be an excellent means for pulse compression and a platform for a rich nonlinear optical dynamics [1-3]. The capabilities of IC-HCPCF in withstanding high laser energy, in engineering its dispersion and in controlling the optical nonlinearities via the choice of the filling gas and its pressure created a new paradigm in the propagation dynamics of HE-USP in gas-filled IC-HCPCF [4]. Notably, in addition to the common nonlinear optical effects such as self-phase-modulation (SPM), four-wave mixing (FWM), stimulated Raman scattering (SRS), gas-filled IC-HCPCF stands out with specific plasma-induced dynamics such as that of "floating" solitons [2]. In [2], a theoretical model treated the case of single mode propagation in an inert gas, and showed that self-compression of USP with ionizing intensities is initiated by the onset of plasma, and is followed by a strong soliton-fission manifested as a 'shower' of hundreds of solitons. In parallel, the use of Raman active gases such as atmospheric air implies the co-existence of plasma blueshift (PBS) and soliton self-frequency redshift (SSFRS), thus opening new and exciting means of controlling the soliton dynamics. Whilst the interplay between PBS and SSFRS has been the subject of several theoretical work, little experimental work has been done so far on the subject [5]. Here, we report on experimental and theoretical work related to the propagation dynamics of an HE-USP in a few meter long air-filled IC-HCPCF. In particular, we show the role of plasma, Raman scattering and IC-HCPCF higher order modes (HOM) in the self-compression of optical pulses with duration of $\sim 600 \mathrm{fs}$ and energy in the range 100-300 $\mu \mathrm{J}$ down to $\sim 20$ fs with little energy loss and in a stable manner. Furthermore, we show, for the first time to our knowledge, experimentally and theoretically soliton spectral oscillation between blueshift and redshift as the input pulse energy is increased.

Fig. 1 summarizes the experimental results. The experiment consists of propagating a 580 fs duration Yb-based laser with energy range of 100-300 $\mu \mathrm{J}$ and central wavelength at $1030 \mathrm{~nm}$ in a 4 meter long Kagome IC-HCPCF exposed to atmospheric pressure. The fiber has a core with negative-curvature contour and an inner diameter of $60 \mu \mathrm{m}$. At the laser wavelength, the transmission loss is $20 \mathrm{~dB} / \mathrm{km}$, the group velocity dispersion (GVD) is $\beta_{2}=-480 \mathrm{fs}^{2} / \mathrm{m}$, the third order dispersion (TOD) is $\beta_{3}=1330 \mathrm{fs} 3 / \mathrm{m}$, and the zero GVD wavelength is $803 \mathrm{~nm}$. Fig. 1a shows the frequencyresolved optical gating (FROG) evolution when the energy is increased from $115 \mu \mathrm{J}$ to $253 \mu \mathrm{J}$. The figure shows a sequence of compression and temporal broadening within the range of 20-100 fs, along with spectral blue and redshift. The FROG and the autocorrelation measurements show striking difference between the input pulse and the output compressed pulse (Fig. 1b). Fig. 1c and Fig. 1d show evolution of the full width at half maximum (FWHM) and central wavelength of the pulse with increasing input energy. In the energy range of 115-150 $\mu \mathrm{J}$ the pulse goes through a continuous compression and red shift from $\sim 100$ fs down to $\sim 20 \mathrm{fs}$ and from $1037 \mathrm{~nm}$ to $1065 \mathrm{~nm}$ respectively. For the range of 150-180 $\mu \mathrm{J}$, the double process of compression and redshift stops before a sudden blueshift back to 1035 $\mathrm{nm}$ for input energy between $180 \mu \mathrm{J}$ and $190 \mu \mathrm{J}$. The blueshift is accomplished with a slight temporal broadening. For the range of 190-250 $\mu \mathrm{J}$, a second sequence of compression and redshift takes place. We attribute this "spectral bouncing" to the interplay of plasma, which induces PBS and intrapulse Raman scattering which is behind SSFRS. The spectral evolution with energy, shown in Fig. 1e, corroborates the above scenario with the redshift of the main peak with increasing energy in the range of 123-180 $\mathrm{J}$, and the growth and blueshift of a peak on the spectrum blueedge. The energy transmission ratio with increasing energy is indicative of a slight material absorption, consistent with the plasma density in the presence of floating solitons. Noteworthy is the high stability of the compressed pulse (Fig. 1f) despite the theoretically predicted sensitivity due to soliton fission. Fig. 2 summarizes the numerical results of the spectral and temporal dynamics of the modes and the spatial and temporal dynamic of the generated plasma for an input energy of $200 \mu \mathrm{J}$. Here the pulse propagation in the IC-HCPCF is numerically solved using the full multimodal nonlinear Schrodinger equation (MMNLSE) including the terms associated to plasma effect, Kerr and Raman in air. Each fiber mode is represented by its own dispersion (gas contribution included) and transverse profile. In the 
present case, we limit to the first $4 L P_{0 \mathrm{~m}}$ modes. The figure shows, from left to right, the evolution with fiber propagation of the modal intensity, the plasma density spatio-temporal distribution and $L P_{01}$ and $L P_{02}$ mode spectrotemporal distribution. In contrast with previous results [2], the soliton fission results in only few dominant pulses. We attribute this to the contribution of the HOM. Plotting the central wavelength and the FWHM of the main soliton pulse shows spectral oscillation and self-compression in qualitative agreement with the experiment. However, discrepancy between numerical and experimental results in the overall temporal structure still remains. Simulation work with better matched parameters such as the modal loss and dispersion or ionisation rate with experiment, and including other types of HOM is ongoing.

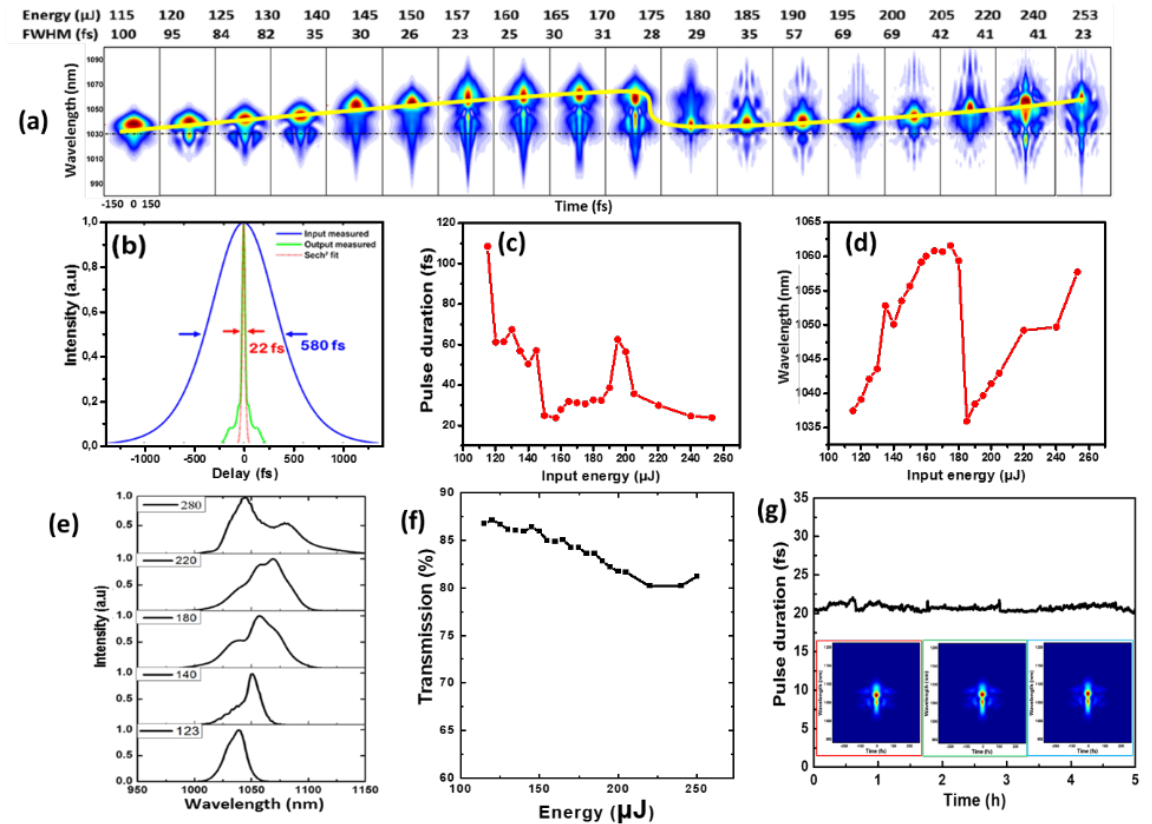

Fig. 1. (a) FROG evolution with input pulse energy. (b) Typical auto-correlation trace of the input pulse (blue) curve and the output pulse (green curve). The red doted curve is a sech2 fit. Evolution with input energy of (c) pulse FWHM, (d) central wavelength, (e) output spectrum and (f) energy transmission coefficient. (g) Time stability of the output pulse FWHM.
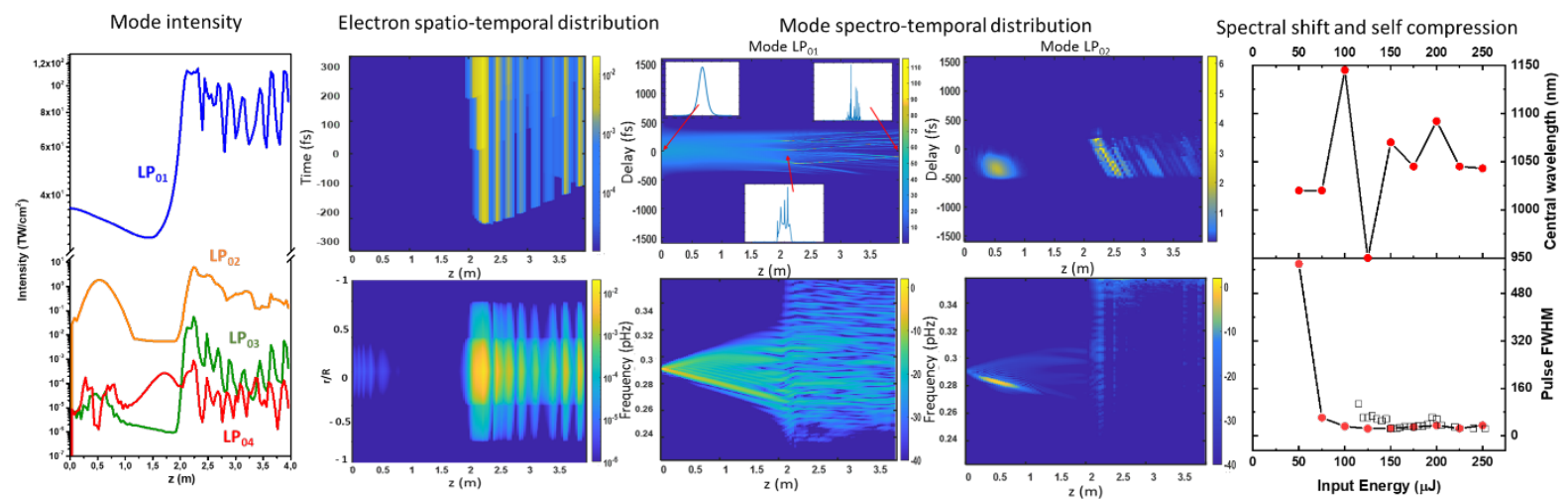

Fig. 2 [Form lhs to rhs] z-evolution of the modal intensities, the electron fractional density temporal (top) and spatial (bottom) distribution, temporal (top) and spectral (bottom) profile of LP 01 ( $3^{\text {rd }}$ column) and LP L (4th column) mode. Last column: central wavelength shift (top) and pulse FWHM (bottom) evolution with energy.

In conclusion, strong and stable self-compression with ionizing intensities, and new spectral-shift dynamics of a $580 \mathrm{fs}$ pulse propagation in air is experimentally observed. The effect are qualitatively reproduced numerically using MMNLSE. Further work is ongoing to investigate the remaining discrepancies between experiment and the numerical model.

\section{References}

[1] T. Balciunas, et al., Nature Communications, 6, N6117, (2015).

[2] M.Saleh et al., J. Opt. 18. pp 013002 (2016).

[3] B.Debord, et al., Photonics Research 7(10), 1134-1141 (2019).
[4] B. Debord et al., Opt. Express, 22(9), 10735-10746 (2014).

[5] M. Maurel, et al., CLEO/Europe-EQEC), Munich, 2019, pp. 